\title{
Dietas artificiais para a criação de larvas e adultos da mosca-das-frutas sul-americana
}

\author{
Adrise Medeiros Nunes(1), Karen Zamboni Costa(2), Kenya Martins Faggioni(2), \\ Maria de Lourdes Zamboni Costa(2), Rafael da Silva Gonçalves ${ }^{(1)}$, Júlio Marcos Melges Walder ${ }^{(2)}$, \\ Mauro Silveira Garcia(1) e Dori Edson Nava(3)
}

\begin{abstract}
(1) Universidade Federal de Pelotas, Faculdade de Agronomia Eliseu Maciel, Departamento de Fitossanidade, Caixa Postal 354 CEP 96010-900 Pelotas, RS. E-mail: adrisenunes@gmail.com, rafaeldasilvagoncalves@gmail.com, garciasmauro@yahoo.com.br (2)Universidade de São Paulo, Centro de Energia Nuclear na Agricultura, Laboratório de Radioentomologia, Avenida Centenário, 303 Caixa Postal 96, CEP 13400-970 Piracicaba, SP. E-mail: karitazc@gmail.com, km.faggioni@gmail.com, lia@cena.usp.br, julio.walder@gmail.com ${ }^{(3)}$ Embrapa Clima Temperado, Laboratório de Entomologia, BR-392, Km 78, Caixa Postal 403, CEP 96001-970 Pelotas, RS. E-mail: dori.edson-nava@embrapa.br
\end{abstract}

Resumo - O objetivo deste trabalho foi adequar as dietas artificiais para o desenvolvimento dos estágios de larva e adulto da mosca-das-frutas sul-americana (Anastrepha fraterculus). Para o estágio larval, foram testadas as seguintes dietas: D1, original, com $10 \mathrm{~g}$ de ágar; D2, modificada, com 3,6 g de ágar; e, D3, modificada, com bagaço seco de cana-de-açúcar. Para os adultos, foram testadas quatro dietas: A, levedura de cerveja + mel (2:1); B, açúcar refinado + extrato de levedura + gérmen de trigo cru (3:1:1); C, extrato de soja + açúcar mascavo + gérmen de trigo cru (3:1:1); e D, levedura seca de cervejaria + mel (2:1). Avaliaram-se os parâmetros biológicos de duração do período ovo-pupa, duração e viabilidade do estágio de pupa, massa média de pupas, razão sexual e duração e viabilidade do período ovo-adulto. O desenvolvimento larval em D1 e D2 foi semelhante e indicou que a criação de larvas pode ser realizada com $1 / 3$ da quantidade de ágar da utilizada em D1. A utilização do bagaço seco de cana-de-açúcar, na dieta artificial, afetou negativamente o desenvolvimento larval. As dietas artificiais com levedura de cerveja + mel e com açúcar refinado + extrato de levedura + gérmen de trigo cru são as mais adequadas para a criação de adultos.

Termos para indexação: Anastrepha fraterculus, controle biológico, criação massal, inseto estéril, Tephritidae.

\section{Artificial diets for the rearing of adult and larvae of South-American fruit fly}

\begin{abstract}
The objective of this work was to adequate artificial diets for the development of larva and adult stages of South-American fruit fly (Anastrepha fraterculus). For the larval stage, the following diets were tested: D1, original diet, with $10 \mathrm{~g}$ agar; D2, modified diet, with $3.6 \mathrm{~g}$ agar; and D3, modified diet, with dry sugarcane bagasse. For adults, four diets were tested: A, brewers' yeast + honey (2:1); B, refined sugar + yeast extract + crude wheat germ (3:1:1); C, soybean extract + brown sugar + crude wheat germ $(3: 1: 1)$; and D, brewers' dried yeast + honey $(2: 1)$. The evaluated biological parameters were: duration of the egg-pupa period, duration and feasibility of the pupa stage, average mass of pupae, sexual ratio, and duration and feasibility of the egg-adult period. Larval stage development for D1 and D2 was similar, and indicated that the larval rearing can be managed with 1/3 agar quantity used in D1. The dry sugarcane bagasse, used in the artificial diet, negatively affected the larval development. Artificial diets with brewers' yeast + honey and with refined sugar + yeast extract + crude wheat germ are the most adequate ones for adult rearing.
\end{abstract}

Index terms: Anastrepha fraterculus, biological control, mass-rearing, steril insect, Tephritidae.

\section{Introdução}

A mosca-das-frutas sul-americana Anastrepha fraterculus (Wiedemann, 1830) (Diptera: Tephritidae) ocorre desde o Sul dos Estados Unidos até o Norte da Argentina (Salles, 1995). No Brasil, é considerada uma das principais pragas de importância econômica, por causar danos diretos, aos frutos, e indiretos em razão das restrições impostas pelos países importadores (Nunes et al., 2012; Bisognin et al., 2013).

Os primeiros estudos com dieta artificial para a mosca-das-frutas sul-americana foram realizados por González (1971), mas foi somente com o trabalho de Salles (1992) que houve avanço na criação, com a

Pesq. agropec. bras., Brasília, v.48, n.10, p.1309-1314, out. 2013 DOI: 10.1590/S0100-204X2013001000001 
utilização de fruto artificial para a obtenção dos ovos e o desenvolvimento de uma dieta artificial para a criação larval. Embora, a técnica tenha propiciado a criação em média escala, o grande entrave à sua utilização para a produção massal está relacionado aos gastos de mão de obra e à baixa fertilidade dos ovos (Salles, 1992). A utilização de painéis de oviposição aumentou a eficiência de obtenção de ovos e, também, propiciou aumento da viabilidade destes (Jaldo et al., 2001).

Em seguida, sucederam-se os trabalhos de Braga Sobrinho et al. (2006), Vera et al. (2007) e Flores et al. (2012), que propuseram mudanças nas técnicas, visando dispor de tecnologia para a criação massal. $\mathrm{Na}$ maioria dos estudos, a dieta artificial básica utilizada foi a de Salles (1992) que, apesar de adequada ao desenvolvimento larval de $A$. fraterculus, apresenta alto custo, em razão, principalmente, do uso do ágar como gelificante. Esse polissacarídeo é a substância preferida para se manter a consistência da dieta, porque é compatível com seus componentes; porém, representa cerca de 60 a $70 \%$ do custo total das dietas artificiais, utilizadas nas criações dos insetos (Parra, 2007). De acordo com Braga Sobrinho et al. (2006), a utilização de produtos locais de baixo custo, como o bagaço de cana-de-açúcar e o farelo de soja, pode viabilizar a criação massal deste inseto e diminuir os custos de produção.

Para a dieta dos adultos, a maioria das biofábricas de moscas-das-frutas de todo o mundo utilizam, como fonte proteica, a levedura hidrolisada Saccharomyces cerevisiae Meyen ex E.C. Hansen, 1883, importada, o que eleva os custos de produção dos insetos (Aluja et al., 2001). A substituição destes ingredientes importados por produtos nacionais pode representar um avanço na criação de mosca-das-frutas, para o controle biológico e a utilização da técnica do inseto estéril (Morelli et al., 2012; Silva Neto et al., 2012).

De acordo com Parker (2005), as dietas podem ser consideradas os componentes mais importantes da criação e, com a mão de obra, constituem os principais custos. Essas mudanças mostram uma evolução das técnicas de criação de $A$. fraterculus e apontam para a viabilidade de sua produção em grande escala (produção massal), desde que adequações sejam feitas em relação à utilização de ingredientes de menor custo.

O objetivo deste trabalho foi readequar a dieta artificial para o desenvolvimento larval e testar diferentes dietas artificiais, para a criação de adultos da mosca-das-frutas sul-americana.

\section{Material e Métodos}

Os experimentos foram realizados no Laboratório de Radioentomologia do Centro de Energia Nuclear na Agricultura (Cena/USP), em Piracicaba, SP, em salas climatizadas a $25 \pm 2^{\circ} \mathrm{C}$, UR $70 \pm 10 \%$ e fotófase de 12 horas. Os insetos foram obtidos da criação de manutenção, realizada em dieta artificial descrita por Salles (1992).

Para avaliar o efeito do gelificante utilizado na dieta artificial para larvas, foram testadas duas modificações: uma na quantidade do agente gelificante; e outra na substituição do gelificante por um texturizante, na dieta artificial proposta por Salles (1992). Foram estabelecidos os seguintes tratamentos com a dieta de Salles (1992): D1, original, com 10 g de ágar; D2, modificada, com 3,6 g de ágar; e, D3, modificada, com substituição do ágar por bagaço seco de cana-de-açúcar triturado e esterilizado, como texturizante (Tabela 1). Para o preparo da dieta artificial, primeiramente, foram colocados em liquidificador os ingredientes sólidos: açúcar refinado, levedura de cerveja liofilizada Brewcell (Biorigin, Lençóis Paulista, SP) e gérmen de trigo cru (Walmon, São Paulo, SP), com $400 \mathrm{~mL}$ de água destilada. Em seguida, os ingredientes foram homogeneizados por $30 \mathrm{~s}$. O metilparahidroxibenzoato Nipagin (Vetec, Química Fina Ltda., Duque de Caxias, RJ) (diluído a 10\% em álcool etílico), o benzoato de sódio da Vetec (dissolvido em 20 mL de água destilada)

Tabela 1. Ingredientes e quantidades utilizadas no preparo de dieta artificial para o desenvolvimento larval de Anastrepha fraterculus.

\begin{tabular}{lccc}
\hline Ingrediente & \multicolumn{3}{c}{ Quantidade $^{(1)}$} \\
\cline { 2 - 4 } & $\mathrm{D} 1$ & $\mathrm{D} 2$ & $\mathrm{D} 3$ \\
\hline Levedura de cerveja (g) & 60,0 & 60,0 & 60,0 \\
Gérmen de trigo (g) & 60,0 & 60,0 & 60,0 \\
Açúcar refinado (g) & 60,0 & 60,0 & 60,0 \\
Ágar (g) & 10,0 & 3,60 & - \\
Bagaço de cana-de-açúcar (g) & - & - & 130,0 \\
Benzoato de sódio (g) & 1,0 & 1,0 & 1,0 \\
Nipagin (metil parabeno) (mL) & 8,0 & 8,0 & 8,0 \\
Ácido clorídrico 37\% (mL) & 6,0 & 6,0 & 6,0 \\
Água destilada (mL) & 800,0 & 800,0 & 800,0 \\
\hline
\end{tabular}

${ }^{(1)}$ Composição para o preparo de $1 \mathrm{~L}$ de dieta artificial. Dieta de Salles (1992): D1, original, com $10 \mathrm{~g}$ de ágar; D2, modificada, com 3,6 g de ágar; D3, modificada, com bagaço seco de cana-de-açúcar. 
e o ácido clorídrico concentrado a 37\% (Synth, Diadema, SP) foram posteriormente acrescentados aos demais ingredientes. Por último, o ágar bacteriológico (Alphatec, Barueri, SP) foi dissolvido em $400 \mathrm{~mL}$ de água destilada e levado ao fogo, tendo-se mexido constantemente até a fervura. Logo em seguida, este ágar foi colocado no liquidificador, junto com os outros ingredientes, e agitado por $2 \mathrm{~min}$, até a perfeita homogeneização. NaD3, utilizou-se, como componente texturizante, o bagaço seco de cana-de-açúcar, o qual foi acrescentado à dieta, depois da homogeneização de todos os ingredientes. As dietas foram distribuídas em recipientes de plástico cilíndrico $(500 \mathrm{~mL})$, com tampa perfurada para permitir a aeração, que receberam $100 \mathrm{~mL}$ de dieta. Para cada recipiente, foi colocado $0,1 \mathrm{~mL}$ de ovos de A. fraterculus (aproximadamente 1.170 ovos) sobre papel filtro.

Após o desenvolvimento larval, as pré-pupas foram separadas da dieta artificial por lavagem em água corrente, tendo-se utilizado uma peneira com malha de 2,0 $\mathrm{mm}$. Em seguida, as pré-pupas foram transferidas para recipientes com vermiculita superfina (tipo GR4 de 0 a $0,9 \mathrm{~mm}$ ) umedecida para a pupação. As pupas permaneceram em vermiculita por 10 dias e, então, foram separadas da vermiculita por peneiramento e acondicionadas no mesmo recipiente até sua emergência.

Foram avaliadas: a duração do período ovo-pupa, a duração e a viabilidade do estágio de pupa, a massa média de pupas, a razão sexual e a duração e viabilidade do período ovo-adulto. Os insetos foram pesados depois de 24 horas da pupação, e a razão sexual (rs) foi determinada pela fórmula rs = fêmea / (fêmea + macho), proposta por Silveira Neto et al. (1976). O delineamento experimental inteiramente casualizado foi utilizado com 10 repetições (potes com dieta artificial, com $0,1 \mathrm{~mL}$ de ovos) para cada tratamento. Os dados foram submetidos à análise de variância, e as médias foram comparadas pelo teste de Tukey, a 5\% de probabilidade, com auxílio do programa SAS, versão 9.2 (SAS Institute, Cary, EUA).

Para avaliar o efeito de dietas, com diferentes fontes proteicas para adultos, foram testadas quatro dietas artificiais, compostas por: A, levedura de cerveja liofilizada (Brewcell) + mel (2:1); B, açúcar refinado (União, São Paulo, SP) + extrato de levedura (Bionis YE MF e YE NS) da Biorigin + gérmen de trigo cru da Walmon (3:1:1); C, extrato de soja (Jasmine, Curitiba,
PR) + açúcar mascavo (MaisVita, São Bernardo do Campo, SP) + gérmen de trigo cru da Walmon (3:1:1); e D, levedura seca de cervejaria Hicell da Biorigin + mel $(2: 1)$.

Vinte e quatro horas após a emergência, 25 casais foram individualizados em gaiolas, feitas a partir de copo de plástico transparente $(700 \mathrm{~mL})$ perfurados, para permitir aeração. $\mathrm{Na}$ parte superior (diâmetro maior), foi colocado tecido voile vermelho, siliconado, que serviu de substrato para a oviposição dos ovos. O silicone foi passado sobre o tecido em uma fina camada, de espessura uniforme (1 mm), com auxílio de um cartão. A atração das fêmeas para o tecido de oviposição foi realizada com a colocação de lâmpadas fluorescentes próximas ao mesmo tecido.

Os casais foram alimentados com as dietas (tratamento) e, também, foi oferecida água destilada, embebida em algodão hidrófilo. Tanto o alimento como a água foram colocados em placas de Petri (3,0 cm de diâmetro) no interior das gaiolas. Foram determinados os parâmetros biológicos, referentes à duração dos períodos de pré-oviposição e oviposição, fecundidade diária e total, longevidade de machos e fêmeas e fertilidade de ovos. A fecundidade foi avaliada diariamente, por meio da contagem do número de ovos, até a morte da fêmea; e a longevidade de machos e fêmeas foi avaliada diariamente durante dois meses.

Após a primeira oviposição, por um período de 10 dias, os ovos de cada tratamento foram retirados diariamente do substrato de oviposição, contados e colocados sobre um tecido de malha preta de algodão e, posteriormente, transferidos para uma bandeja de plástico $(14 \times 21 \mathrm{~cm})$, com um "pano-esponja vegetal" $(17 \times 19 \mathrm{~cm})$ da Bettanin, umedecido e, depois de 72 horas, a fertilidade dos ovos foi avaliada.

O delineamento experimental inteiramente casualizado foi utilizado, com 25 repetições (casais) para cada tratamento; os dados foram submetidos à análise de variância, e as médias foram comparadas pelo teste de Tukey, a 5\% de probabilidade, com auxílio do programa SAS, versão 9.2.

\section{Resultados e Discussão}

Os valores dos parâmetros biológicos avaliados para as dietas D1 e D2 não diferiram entre si, o que indica que a redução da quantidade de ágar não afetou o desenvolvimento larval de $A$. fraterculus (Tabela 2). 
Entretanto, a substituição do ágar pelo bagaço seco de cana-de-açúcar (D3) afetou negativamente os parâmetros biológicos avaliados. Assim, a duração do período ovo-pupa de $A$. fraterculus criada nas dietas D1 e D2, com 10 e 3,6 g de ágar, respectivamente, foi maior do que o valor registrado na dieta com bagaço seco de cana-de-açúcar. Insetos criados nas dietas D1 e D2 não apresentaram diferença significativa na duração do estágio de pupa, enquanto os criados na dieta D3 diferiram somente de D1 (Tabela 2). González (1971) relatou duração do estágio de larva de 8 dias e do estágio de pupa de 12 dias para $A$. fraterculus, criados com dieta artificial descrita por Finney (1956), em que não foi utilizado ágar como componente gelificante.

A substituição do ágar pelo bagaço seco de cana-de-açúcar reduziu a massa e a viabilidade de pupa. Pupas oriundas de larvas alimentadas com as dietas D1 e D2 tiveram maior massa do que as oriundas de larvas alimentadas com a dieta D3 e apresentaram maior viabilidade pupal. Os valores registrados para a massa de pupa, nas dietas D1 e D2, foram mais elevados do que os obtidos por González (1971) (13,0 mg) e Vera et al. (2007) (13,1 mg), porém, inferiores aos observados (18,0 mg) por Jaldo et al. (2001). Provavelmente, um dos fatores que contribuiu para a baixa viabilidade de pupa, na dieta D3, além da falta de consistência do meio, que pode interferir na locomoção e alimentação das larvas, é o fato de que, no final do terceiro instar (pré-pupas), estas foram lavadas em água corrente, para retirada da dieta artificial. A presença do bagaço seco de cana-de-açúcar também pode ter danificado fisicamente as larvas e diminuído sua viabilidade.

A razão sexual também foi afetada, quando se substituiu o ágar pelo bagaço seco de cana-de-açúcar, tendo-se observado na dieta D3 um menor número de fêmeas (Tabela 2). Valores com razão sexual próxima aos obtidos nas dietas D1 e D2 foram relatados por Jaldo et al. (2001) $(0,50)$ e Vera et al. (2007) $(0,53)$, em estudos que avaliaram a qualidade dos insetos produzidos ao longo dos anos em laboratório.

A viabilidade do período ovo-adulto foi maior na dieta D2. A dieta D1 apresentou viabilidade de 17,55\%, a qual diferiu de D3 (1,91\%). Jaldo et al. (2001) e Vera et al. (2007) relataram viabilidade de larva-adulto de 44,0 e 56,3, respectivamente. Assim, de acordo com os resultados obtidos no presente trabalho, a criação de A. fraterculus pode ser realizada com $1 / 3$ da quantidade proposta na dieta de Salles (1992), sem que ocorra perda de qualidade dos insetos produzidos e, assim, com possibilidade de redução do custo da dieta.

Em relação às dietas testadas para adultos de A. fraterculus, observa-se que a duração do período de pré-oviposição não diferiu entre as quatro dietas e variou de 10,42 a 11,75 dias (Tabela 3). Em trabalho realizado por González (1971), com adultos alimentados com dieta composta por açúcar e extrato de soja (4:1), o período de pré-oviposição foi de 8 dias.

Para o período de oviposição, os insetos alimentados com as dietas A, B e C ovipositaram por um período maior do que os alimentados com a dieta $\mathrm{D}$, porém não houve diferença entre os tratamentos. O período de oviposição obtido por González (1971) foi de 20 dias, o qual foi maior do que encontrado no presente trabalho.

A fecundidade média diária das fêmeas alimentadas com as dietas A e B foram semelhantes e superiores à das dietas $\mathrm{C}$ e $\mathrm{D}$, o que mostrou que estas foram nutricionalmente mais adequadas para os adultos de A. fraterculus (Tabela 3). Salles (1992) utilizou

Tabela 2. Média \pm erro-padrão dos parâmetros biológicos de Anastrepha fraterculus, criada em dieta artificial, com diferentes quantidades de ágar e bagaço seco de cana-de-açúcar, em condições de temperatura de $25 \pm 2^{\circ} \mathrm{C}$, UR de $70 \pm 10 \%$ e fotófase de 12 horas $^{(1)}$.

\begin{tabular}{lcccc}
\hline Parâmetros biológicos & Dieta $1^{(2)}$ & Dieta 2 & Dieta 3 & Valor P \\
\hline Duração do período ovo-pupa (dias) & $11,50 \pm 0,17 \mathrm{a}$ & $11,30 \pm 0,15 \mathrm{a}$ & $10,00 \pm 0,00 \mathrm{~b}$ & 38,93 \\
Duração do estágio de pupa (dias) & $12,70 \pm 0,21 \mathrm{a}$ & $12,50 \pm 0,17 \mathrm{ab}$ & $12,00 \pm 0,00 \mathrm{~b}$ & 5,32 \\
Massa média de pupa (mg) & $15,42 \pm 0,15 \mathrm{a}$ & $15,33 \pm 0,20 \mathrm{a}$ & $10,26 \pm 1,76 \mathrm{~b}$ & 8,25 \\
Viabilidade de pupa (\%) & $76,70 \pm 2,10 \mathrm{a}$ & $88,70 \pm 1,31 \mathrm{a}$ & $40,60 \pm 8,04 \mathrm{~b}$ & 26,57 \\
Duração do período ovo-adulto (dias) & $24,20 \pm 0,33 \mathrm{a}$ & $23,80 \pm 0,25 \mathrm{a}$ & $22,00 \pm 0,00 \mathrm{~b}$ & 0,0113 \\
Razão sexual & $0,51 \pm 0,017 \mathrm{a}$ & $0,53 \pm 0,010 \mathrm{a}$ & $0,33 \pm 0,061 \mathrm{~b}$ & 0,0016 \\
Viabilidade ovo-adulto (\%) & $17,55 \pm 1,99 \mathrm{~b}$ & $26,20 \pm 2,41 \mathrm{a}$ & $1,91 \pm 1,50 \mathrm{c}$ & 8,88 \\
\hline
\end{tabular}

${ }^{(1)}$ Médias seguidas de letras iguais, nas linhas, não diferem entre si, pelo teste de Tukey, a 5\% de probabilidade. ${ }^{(2)}$ Dieta de Salles (1992): D1, original, com $10 \mathrm{~g}$ de ágar; D2, modificada, com 3,6 g de ágar; e D3, modificada, com substituição do ágar por bagaço seco de cana-de-açúcar como texturizante. 
Tabela 3. Média \pm erro-padrão dos parâmetros biológicos de Anastrepha fraterculus, alimentada com diferentes dietas artificiais para adulto, em condições de temperatura de $25 \pm 2^{\circ} \mathrm{C}$, UR de $70 \pm 10 \%$ e fotófase de 12 horas ${ }^{(1)}$.

\begin{tabular}{|c|c|c|c|c|c|c|}
\hline \multirow[t]{2}{*}{ Parâmetros biológicos } & \multicolumn{4}{|c|}{$\operatorname{Dietas}^{(2)}$} & \multirow[t]{2}{*}{ Valor F } & \multirow[t]{2}{*}{ Valor $\mathrm{p}$} \\
\hline & A & $\mathrm{B}$ & $\mathrm{C}$ & $\mathrm{D}$ & & \\
\hline Duração do período de pré-oviposição (dias) & $10,82 \pm 0,55 \mathrm{a}$ & $10,95 \pm 0,41 \mathrm{a}$ & $10,42 \pm 0,29 \mathrm{a}$ & $11,75 \pm 1,24 \mathrm{a}$ & 0,56 & 0,6402 \\
\hline Duração do período de oviposição (dias) & $15,27 \pm 1,54 \mathrm{a}$ & $13,27 \pm 1,85 \mathrm{a}$ & $15,42 \pm 1,43 a$ & $9,08 \pm 1,87 \mathrm{a}$ & 2,18 & 0,0992 \\
\hline Fecundidade diária & $21,96 \pm 1,54 \mathrm{ab}$ & $30,17 \pm 2,54 a$ & $18,79 \pm 1,80 \mathrm{~b}$ & $20,14 \pm 2,92 b$ & 5,40 & 0,0022 \\
\hline Fecundidade total & $343,86 \pm 42,48 \mathrm{ab}$ & $449,32 \pm 74,62 \mathrm{a}$ & $251,08 \pm 20,15 \mathrm{ab}$ & $201,25 \pm 61,77 b$ & 3,17 & 0,0303 \\
\hline Longevidade de machos (dias) & $25,14 \pm 1,57 \mathrm{a}$ & $24,63 \pm 4,28 \mathrm{a}$ & $21,67 \pm 2,37 \mathrm{a}$ & $24,36 \pm 3,51 \mathrm{a}$ & 0,47 & 0,7055 \\
\hline Longevidade de fêmeas (dias) & $30,59 \pm 2,15 \mathrm{a}$ & $29,64 \pm 2,66 \mathrm{a}$ & $28,28 \pm 2,49 \mathrm{a}$ & $22,81 \pm 2,23 \mathrm{a}$ & 1,85 & 0,1462 \\
\hline Fertilidade de ovos (\%) & $86,60 \pm 1,36 a$ & $80,70 \pm 1,24 \mathrm{ab}$ & $82,60 \pm 3,10 \mathrm{a}$ & $72,20 \pm 3,20 \mathrm{~b}$ & 6,35 & 0,0014 \\
\hline
\end{tabular}

${ }^{(1)}$ Médias seguidas de letras iguais, nas linhas, não diferem entre si, pelo teste de Tukey, a 5\% de probabilidade. (2)Dietas: A, levedura de cerveja + mel (2:1); B, açúcar refinado + extrato de levedura (Bionis YE MF e YE NS) + gérmen de trigo cru à proporção de (3:1:1); C, extrato de soja + açúcar mascavo + gérmen de trigo cru (3:1:1); e D, levedura seca de cervejaria Hicell + mel (2:1).

proteína hidrolisada de milho e açúcar mascavo, para a alimentação dos adultos, e obteve fecundidade total de 394 ovos, número próximo ao obtido no presente trabalho para a dieta A (344 ovos) e inferior ao obtido para a dieta B (449 ovos). Jaldo et al. (2001) relataram fecundidade de 625 ovos, em experimentos em que utilizaram como alimento para os adultos uma dieta composta de proteína hidrolisada importada, proteína hidrolisada de milho, açúcar refinado, água, vitaminas (Dayamineral, Abbott) e aminoácidos (Aminocefa 5\%, Roux Ocefa). O total de ovos observado por Jaldo et al. (2001) foi maior do que o obtido no presente trabalho, porém a proteína hidrolisada utilizada foi importada, o que implica em alto custo de produção. Braga Sobrinho et al. (2006) testaram diferentes dietas para adultos e obtiveram os melhores resultados com a dieta composta por proteína hidrolisada de milho, hidrolisado enzimático de fermento e açúcar cristalizado à proporção de $(3: 1: 3)$, em que a fecundidade média diária foi de 16,34 ovos; valores superiores foram encontrados no presente trabalho.

As dietas utilizadas para alimentação dos adultos não influenciaram a longevidade de machos e de fêmeas, que variou de 21,67 a 24,63 e de 22,81 a 30,59 dias, respectivamente (Tabela 3). A viabilidade do período embrionário, para insetos alimentados com as dietas A, B e C, foi superior ao registrado na dieta D, a qual foi significativamente igual à dieta $\mathrm{B}$. Com relação à fertilidade dos ovos, os valores encontrados no presente trabalho foram superiores aos obtidos por González (1971), que observou fertilidade de $45 \%$, e por Braga Sobrinho et al. (2006), que observaram 60\% na melhor dieta (Tabela 3). De acordo com Morelli et al. (2012) e Silva Neto et al. (2012), os quais utilizaram fontes proteicas oriundas de produtos comerciais nacionais (Bionis), foram observados melhores resultados nos parâmetros biológicos avaliados do que os obtidos com a fonte proteica importada, com diminuição em mais de $80 \%$ no custo de produção. Assim, verifica-se que as dietas $\mathrm{A}$ e $\mathrm{B}$ à base de extrato de levedura são adequadas para a criação dos insetos adultos.

\section{Conclusões}

1. A criação de larvas de Anastrepha fraterculus pode ser realizada com $1 / 3$ da quantidade de ágar descrita na dieta original.

2. A substituição do ágar pelo bagaço seco de cana-de-açúcar afeta negativamente a maioria dos parâmetros biológicos de $A$. fraterculus.

3. As dietas à base de levedura de cerveja + mel (2:1) e açúcar refinado + extrato de levedura + gérmen de trigo cru (3:1:1) são as mais adequadas para o estágio adulto de $A$. fraterculus.

\section{Referências}

ALUJA, M.; JÁCOME, I.; MACIAS-ORDÓÑEZ, R. Effect of adult nutrition on male sexual performance in four tropical fruit fly species of the genus Anastrepha (Diptera: Tephritidae). Journal of Insect Behavior, v.14, p.759-775, 2001. DOI: 10.1023/A:1013037400676.

BISOGNIN, M.; NAVA, D.E.; LISBÔA, H.; BISOGNIN, A.Z.; GARCIA, M.S.; VALGAS, R.A.; DIEZ-RODRÍGUEZ, G.I.; BOTTON, M.; ANTUNES, L.E.C. Biologia da mosca-das-frutas sul-americana em frutos de mirtilo, amoreira-preta, araçazeiro e pitangueira. Pesquisa Agropecuária Brasileira, v.48, p.141-147, 2013. DOI: 10.1590/S0100-204X2013000200003. 
BRAGA SOBRINHO, R.; CACERES, C.; ISLAM, A.; WORNOAYPORN, V.; ENKERLIM, W. Improving mass rearing technology for South American fruit fly (Diptera: Thephritidae). Revista Caatinga, v.19, p.310-316, 2006.

FINNEY, G.L. A fortifield carrot medium for mass culture of the Oriental fruit fly and certain other Tephritidae. Journal of Economic Entomology, v.49, p.134-136, 1956.

FLORES, H.S.; HERNÁNDEZ, E.; TOLEDO J. Dessarrollo de un sistema de cría artificial para Anastrepha fraterculus (Wied.) (Diptera: Tephritidae). Acta Zoológica Mexicana, v.28, p.321-340, 2012.

GONZÁLEZ, J.B.; VARGAS, C.V.; JARA, B.P. Estudios sobre la aplicación de la técnica de machos estériles en el control de la mosca sudamericana de la fruta, Anastrepha fraterculus (Wied.). Revista Peruana de Entomología, v.14, p.66-86, 1971.

JALDO H.E.; GRAMAJO, M.C.; WILLINK, E. Mass rearing of Anastrepha fraterculus (Diptera: Tephritidae): a preliminary strategy. Florida Entomologist, v.84, p.716-718, 2001. DOI: $10.2307 / 3496407$.

MORELLI, R.; COSTA, K.Z.; FAGIONI, K.M.; COSTA, M.L.Z.; NASCIMENTO, A.; PIMENTEL, R.M.A.; WALDER, J.M.M. New protein sources in adults diet for mass-rearing of Anastrepha fraterculus (Diptera:Tephritidae). Brazilian Archives of Biology and Technology, v.55, p.827-833, 2012. DOI: 10.1590/ S1516-89132012000600004.

NUNES, A.M.; MÜLLER, F.A.; GONÇALVES, R. da S.; GARCIA, M.S.; COSTA, V.A.; NAVA, D.E. Moscas frugívoras e seus parasitoides nos municípios de Pelotas e Capão do Leão, Rio Grande do Sul, Brasil. Ciência Rural, v.42, p.6-12, 2012. DOI: 10.1590/S0103-84782012000100002.
PARKER, A.G. Mass-rearing for sterile insect release. In: DYCK, V.A.; HENDRICHS, J.; ROBINSON, A.S. (Ed.). Sterile insect technique: principles and practice in area-wide integrated pest management. Dordrecht: Springer, 2005. p.209-232. DOI: 10.1007/1-4020-4051-2_8.

PARRA, J.R.P. Exigências físicas, químicas e biológicas para alimentação. In: PARRA, J.R.P. (Ed.). Técnicas de criação de insetos para programas de controle biológico. Piracicaba: FEALQ, 2007. 134p.

SALLES, L.A.B. Bioecologia e controle da mosca-das-frutas sul-americana. Pelotas: Embrapa Clima Temperado, 1995. 58p.

SALLES, L.A.B. Metodologia de criação de Anastrepha fraterculus (Wiedemann, 1830) (Diptera: Tephritidae) em dieta artificial em laboratório. Anais da Sociedade Entomológica do Brasil, v.21, p.479-486, 1992.

SILVA NETO, A.M. da; SANTOS, T.R. de O.; DIAS, V.S.; JOACHIM-BRAVO, I.S.; BENEVIDES, L. de J.; BENEVIDES, C.M. de J.; SILVA, M.V.L.; SANTOS, D.C.C. dos; VIRGÍNIO, J.; OLIVEIRA, G.B.; WALDER, J.M.M.; PARANHOS, B.A.J.; NASCIMENTO, A.S. do. Mass-rearing of Mediterranean fruit fly using low-cost yeast products produced in Brazil. Scientia Agricola, v.69, p.364-369, 2012. DOI: 10.1590/ S0103-90162012000600004.

SILVEIRA NETO, S.; NAKANO, O.; BARBIN, D.; VILLA-NOVA, N.A. Manual de ecologia dos insetos. Piracicaba: Agronômica Ceres, 1976. 419p.

VERA, M.T.; ABRAHAM, S.; OVIEDO, A.; WILLINK, E. Demographic and quality control parameters of Anastrepha fraterculus (Diptera: Tephritidae) maintained under artificial rearing. Florida Entomologist, v.90, p.53-57, 2007. DOI: 10.1653/0015-4040(2007)90[53:DAQCPO]2.0.CO;2.

$\overline{\text { Recebido em } 2 \text { de abril de } 2013 \text { e aprovado em } 30 \text { de setembro de } 2013}$ 